\title{
Validation of Updated Partin's Table in Pakistani Patients undergoing Radical Prostatectomy for Prostate Cancer
}

Syed M Nazim ${ }^{1 *}$, Farhat Abbas ${ }^{1}$, Nuzhat Faruqui' Muhammad Islam² and Zubair Ahmad ${ }^{3}$

${ }^{1}$ Departments of Surgery, Aga Khan University, Karachi, Pakistan

${ }^{2}$ Community Health Sciences, Aga Khan University, Karachi, Pakistan

${ }^{3}$ Pathology \& Microbiology, Aga Khan University, Karachi, Pakistan

\begin{abstract}
Objectives: To establish the usefulness and validity of 2007 Partin's table in our population with prostate cancer.

Materials and methods: Between January 1998 to June 2009, all patients with clinically localized carcinoma prostate who were treated with intent of radical retro-pubic prostatectomy (RRP) were included. Clinical, operative and pathological data was gathered. All biopsy and final histopathology Gleason scores were re-assigned in a double blind manner. Pre-operative serum PSA, TNM clinical stage and biopsy Gleason scores were plotted on Partin's table and its predictive value and pathological findings of specimen were compared and analyzed by using Receiver operating characteristic (ROC) analysis.

Results: A total of 109 of 138 patients were included in final analysis. The median age was $65 \pm 5.8$ years. The pre-operative serum PSA values and clinical stages were higher in our cohort of patients as compared to Partin's cohort. At pathological assessment of resected specimen, organ confined disease was present in $58 \%$ of patients, seminal vesicles were involved in $22 \%$ and lymph node metastasis was present in $12 \%$ of patients. The accuracy of Partin's table derived probability was high with area under curve (AUC) of 0.82 for organ confinement, 0.805 for seminal vesicle involvement and 0.714 for lymph node involvement respectively.
\end{abstract}

Conclusions: The 2007 Partin's table has a reasonably high predictive value for the final histo-pathological features. This predictive model can be used in Pakistani patients with carcinoma prostate with comparable accuracy.

Keyeords: Partin's Table; Prostate cancer; Radical prostatectomy; Validation; Pakistan

\section{Introduction}

Prostate cancer is a disease of increasing significance worldwide and one of the most common cancers and leading causes of death in industrialized 1nations [1]. Localized or organ confined disease offers the best chance of cure and therefore in patients with this disease, it is critical to have an accurate prediction of the final pathological stage, so that the appropriate therapy can be given [2]. The treatment options for localized prostate cancer include radical prostatectomy, external beam radiation therapy or brachytherapy, active surveillance or hormonal therapy [3]. Radical prostatectomy (RP) is the treatment which is known to be most effective for the localized disease [4,5].

Several nomograms have been developed to predict the final pathological stage as no single clinical test or examination finding can accurately predict this. Among these, Partin's table is most widely used in clinics worldwide. This table was developed from a selective group of patients with localized prostate cancer in U.S. \& can estimate the pathological stage from clinical parameters like pre-treatment PSA level, clinical stage and biopsy Gleason score [6]. It therefore helps urologists, radiation oncologists, medical oncologists and general practitioners in predicting the expected disease stage ranging from organ confined status to lymph node metastasis, if the prostate is removed surgically [7].

Although this model has been both internally and externally validated, there are always concerns whether it can be applied to the patient population outside U.S. The biological behavior of prostate cancer differs between the Asian and Western populations [2]. Also, there are differences in screening, selection and treatment protocols.
The predictive value of Partin's table has not been established for Pakistani population therefore, in this study, we evaluated the validity of current Partin table updated in 2007 in a subset of surgically treated patients with clinically localized prostate cancer.

\section{Materials and Methods}

This was a retrospective analysis of data over a period of 12 years from Jan 1998- June 2009. The clinical and pathologic data of patients who had bilateral pelvic lymph node dissection with intent to treat by radical prostatectomy for localized prostate cancer by a single surgeon (FA) at the Aga Khan University and hospital were reviewed. Patients with incomplete/ missing data, those whose biopsies were done outside our hospital and were not available for review or who had neo-adjuvant hormonal or radiation treatment were excluded. The final study population consisted of 109 patients. The pre-operative clinical and pathological data included serum PSA level (within 6 weeks prior to the surgery), the clinical stage as determined by digital rectal examination

*Corresponding author: Farhat Abbas, MD, FCPS, FRCSEd, FRCS, FEBU, FACS, The Hussein. Cumber, Professor (Urology), Aga Khan University, Stadium Road, PO Box 3500, Karachi-74800, Pakistan, Tel: (92) 2134864402 / 34864409 ; Fax: (92) 21 34934294; E-mail: farhat.abbas@aku.edu

Received September 28, 2010; Accepted November 02, 2011; Published November 04, 2011

Citation: Nazim SM, Abbas F, Faruqui N, Islam M, Ahmad Z (2011) Validation of Updated Partin's Table in Pakistani Patients undergoing Radical Prostatectomy for Prostate Cancer J Cancer Sci Ther S1. doi:10.4172/1948-5956.S1-010

Copyright: (c) 2011 Nazim SM, et al. This is an open-access article distributed under the terms of the Creative Commons Attribution License, which permits unrestricted use, distribution, and reproduction in any medium, provided the original author and source are credited. 
and was assigned according to 2002 AJCC staging system and biopsy Gleason score. All patients had ultrasound guided systemic sextant (six cores) or octant (eight core) biopsy done. Both the biopsy slides and prostatectomy specimen slides were reviewed by a single dedicated histo-pathologist (ZA) in a double blinded manner and these were graded histologically using Gleason scoring system according to the 2005 ISUP consensus.

The final pathological stage was categorized in each patient as outlined in Partin's table. This included gradual progression of disease as follows; organ confined disease (OC) as long as prostatic capsule was not breached by tumor, extra-prostatic extension (EPE) if tumor reached the inked surface i.e. surgical margin was positive, Seminal vesicle invasion (SVI) if patient had invasion of seminal vesicles without lymph node involvement and lymph node involvement (LNI) if patient has lymph node involvement.

All statistical analyses were performed on a commercially available SPSS software package. The 2007 updated Partin's table was applied to our patients and based on the pre-operative parameters (PSA, Clinical stage, and Gleason grade) the prediction of organ confinement, seminal vesicle and lymph node involvement was calculated. Sensitivity and specificity of Partin tables was calculated and Receiver operating characteristics (ROC) analysis was done to assess the discriminative ability of this table in our patient population.

\section{Results}

A total of 138 patients were operated with the intent of radical retropubic prostatectomy (RRP) during the study period, of which 109 (79\%) fulfilling the criteria were included in final analysis. 90 patients (83\%) had RRP with bilateral negative pelvic lymph node dissection (PLND). In 7 patients with gross lymph node metastases on frozen section examination, only bilateral orchidectomy was done while in 12 patients with microscopic metastases, RRP with bilateral orchidectomy was carried out. The median age at presentation was $65 \pm 5.83$ (49-76) years. The clinical and pathological features i.e. pre operative serum PSA, biopsy Gleason scores and preoperative clinical stages were compared with 2007 Partin's cohort and are shown in Table 1.

The median pre-operative PSA value was $10.5+/-6.3 \mathrm{ng} / \mathrm{ml}(1.5-$ 58.5) (Abbot Hybritech Assay) and it was higher in our cohort of patients as compare to Partin's cohort. Only $5 \%$ of patients in our cohort had serum PSA values less than $4 \mathrm{ng} / \mathrm{ml}$ as compare to $25 \%$ of patients in Partin's cohort. A very high proportion (> 50\%) in our population had PSA values $>10 \mathrm{ng} / \mathrm{ml}$ while Partin's cohort had only $12 \%$ of patients in this range of PSA. $44 \%$ of patients in our study group and $64 \%$ in Partin's cohort had serum PSA value in gray scale i.e. b/w $4.0-10.0 \mathrm{ng} / \mathrm{ml}$.

The distribution of clinical stage showed a remarkable difference. In Partin's cohort, $77 \%$ of patients had T1c disease and only $6 \%$ had $\mathrm{T} 2 \mathrm{~b} / \mathrm{T} 2 \mathrm{c}$ disease. Our cohort showed $30 \%$ and $47 \%$ respectively. The median Gleason score was $6+/-2.4$ (2-10). Patients in our cohort had higher Gleason score with $11 \%$ having least favorable disease (Gleason 8 or $>$ ) as compared to only $3 \%$ in Partin's group. Partin's cohort had $77 \%$ of patients with low grade disease (Gleason 6 or $<$ ) as compare to $58 \%$ of our patients.

Final histo-pathological assessment showed that $58 \%$ of patients had organ confined disease as compared to $73 \%$ in Partin's cohort.

\begin{tabular}{|c|c|c|}
\hline & $\begin{array}{l}\text { Pakistan } 2009 \\
(n=109)\end{array}$ & $\begin{array}{l}\text { Partin 2007* } \\
(n=5730)\end{array}$ \\
\hline \multicolumn{3}{|l|}{ CLINICAL STAGE } \\
\hline T1c & $33(30)$ & $4419(77)$ \\
\hline $\mathrm{T} 2 \mathrm{a}$ & $25(23)$ & 998(17) \\
\hline $\mathrm{T} 2 \mathrm{~b} / \mathrm{T} 2 \mathrm{c}$ & $51(47)$ & $313(6)$ \\
\hline \multicolumn{3}{|l|}{$\mathrm{PSA}(\mathrm{ng} / \mathrm{ml}) \Delta$} \\
\hline $0-2.5$ & $3(3)$ & $452(8)$ \\
\hline $2.6-4.0$ & $2(2)$ & 946 (17) \\
\hline 4.1-6.0 & $15(14)$ & $1994(35)$ \\
\hline $6.1-10.0$ & $34(31)$ & $1671(29)$ \\
\hline$>10.0$ & $55(50)$ & $667(12)$ \\
\hline \multicolumn{3}{|l|}{ BIOPSY GLEASON SUM } \\
\hline $5-6$ & $63(58)$ & $4402(77)$ \\
\hline $3+4=7$ & $23(21)$ & $816(14)$ \\
\hline $4+3=7$ & $11(10)$ & $348(6)$ \\
\hline 8-10 & $12(11)$ & $164(3)$ \\
\hline \multicolumn{3}{|l|}{ PATHOLOGICAL STAGE } \\
\hline Organ confined & $63(58)$ & $4204(73)$ \\
\hline Extra prostatic extension & $9(8)$ & $1276(22)$ \\
\hline Seminal vesicle involvement & $24(22)$ & $180(3)$ \\
\hline Lymph node metastasis & $13(12)$ & $70(1)$ \\
\hline
\end{tabular}

* Makarov et al. [13].

$\triangle$ Abbot Hybritech Assay

Table 1: Comparison of Clinical and pathological features.

\begin{tabular}{|l|l|}
\hline Gleason final Histo-pathology & $\mathrm{N}(\%)^{\star *}$ \\
\hline Same & $65(63.7)$ \\
\hline Up grade & $22(21.6)$ \\
\hline Down grade & $15(14.7)$ \\
\hline
\end{tabular}

** Patients with PLND \& orchidectomy were excluded [7]

Table 2: Comparisons of Biopsy Gleason score and final histopathology Gleason scores.

A much higher proportion of our patients had seminal vesicle involvement and lymph node metastases i.e. $22 \%$ and $12 \%$, while only $3 \%$ and $1 \%$ had that in Partin's cohort, respectively.

A comparison of biopsy Gleason and final histopathology Gleason showed up grading in $21 \%$ of patients while $64 \%$ had similar grade (Table 2).

The accuracy and discriminative ability of Partin tables were assessed using ROC analyses. The AUC for organ confined disease was 0.82 (95\% CI 0.742-0.897), for seminal vesicle involvement 0.80 (95\% CI $0.717-0.893)$ and that for lymph node involvement was 0.714 (CI 0.6-0.83) (Figures 1-3).

\section{Discussion}

Prostate cancer is the $2^{\text {nd }}$ most common cancer in men and fifth most common cancer in the world [1]. Early detection and appropriate treatment can reduce prostate cancer related morbidity and mortality. Many nomograms have been developed for providing diagnostic, staging and prognostic information in patients with prostate cancer [9]. Oesterling et al. [10] in 1987 reported a model including the combination of pre operative variables like clinical stage, serum acid phosphatase and pre operative Gleason score to predict the final pathological stage in patients with clinically localized prostate cancer as compare to individual variables alone. The pathological stage of disease 
determines the final choice of treatment. Partin tables were designed to provide the pathological stage predictions and to guide treatment decisions.

The validation studies of former Partin tables (1997 and 2001) have confirmed its accuracy not only for United States but also for Europe and Asian cohorts $[2,11,12]$ and showed that these tables can be applied to other population cohorts as well. The Partin's table was updated in 2007 by Makarove et al. [13] and its accuracy and discriminative properties have been questioned in various external validation studies $[7,14,15]$. These studies have shown poor relationship between predicted probabilities and observed rates and demonstrated worse performance in populations other than U.S.

Naito et al. [2] suggested that as it takes into account the ethnic differences, variation in prostate gland sizes and biological behavior of the disease, a nomogram based on population of interest is expected to predict the pathological stage better than one developed in an

\section{ROC Curve}

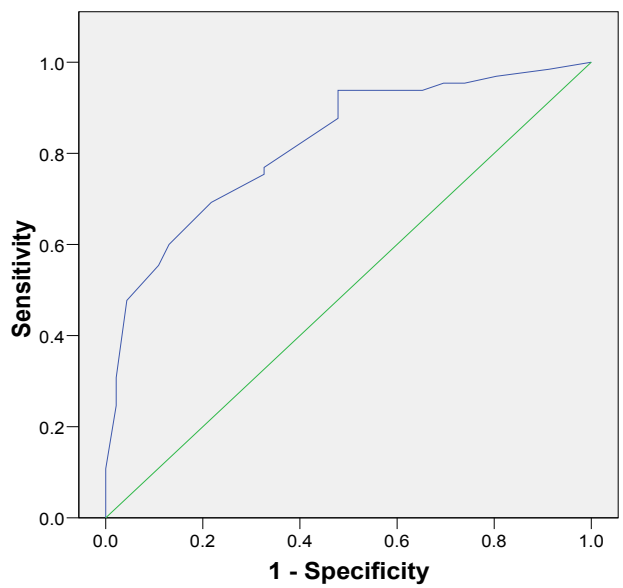

Figure 1: ROC analysis for predicted probabilities of organ confinement.

ROC Curve

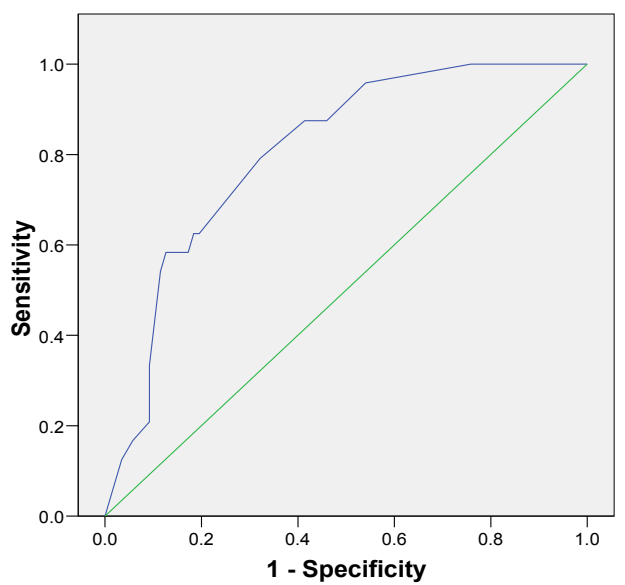

Figure 2: ROC analysis for predicted probabilities of seminal vesicle involvement.

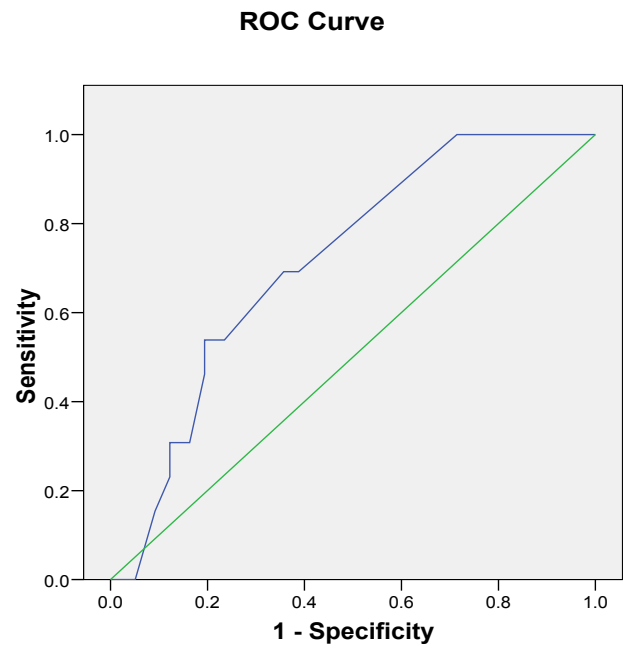

Figure 3: ROC analysis for predicted probabilities of lymph node involvement.

outside population. Ethenic differences account for marked variation in prostate cancer incidence and mortality rates with very high rates amongst African Americans and lowest rates amongst men from South East Asia and China [16,17].

Our study cohorts and Partin's cohort are quite different in terms of the extent and nature of disease. Only $30 \%$ of our study patients compared with $77 \%$ in Partin's series had clinical stage T1c disease [13]. This is due to delayed diagnosis and lack of implementation of early detection methodologies for prostate cancer in our population. More than half of our patients had serum PSA levels $>10 \mathrm{ng} / \mathrm{ml}$ compared to only $12 \%$ in Partin's cohort. Despite higher clinical stage and PSA levels at diagnosis, $58 \%$ of our patients had pathologically organ confined disease as compare to 73 \% in Partin's cohort. $22 \%$ of our patients had seminal vesicle involvement and $12 \%$ had lymph node metastases. In the recent Partin's table [13], the prevalence of seminal vesicle and lymph node involvement was $3 \%$ and $1 \%$ respectively.

Pelvic lymph node dissection (PLND) is the most accurate and reliable staging procedure for the detection of lymph node invasion in prostate cancer [18]. There is still a debate about the extent of dissection i.e. limited vs. extended and in which patients it should be done [19]. There is no prospective randomized clinical trial which has tested the impact of PLND on prostate cancer outcome. Limited PLND may be associated with high rate of false negative findings and it is now recommended to have more extended PLND in order to have a more accurate assessment of lymph nodes and nodal metastases. Briganti et al. [20] and Studer and Collette [21] have reported a high prevalence of lymph node metastasis exceeding $10 \%$ when extended PLND is performed and therefore the updated Partin tables will be limited in predicting lymph node involvement in cohort of patients who undergo extensive PLND. For our patients, we performed limited pelvic lymph node dissection for staging purposes only.

Area under curve (AUC) values has been assessed previously for evaluating the predictability of Partin tables in external validation studies $[11,12,14,15]$. The ideal predictions are donated with $100 \%$ accuracy and values of $50 \%$ or less indicates that the model cannot predict the desired outcome but rather describes the only random 
predictions. Therefore, values of less than $70 \%$ may be considered as a poor result.

Despite the apparent differences in pre-operative variables of our cohort and the derivation cohort of updated Partin's table, the predicted accuracy for the final pathological status was good. Our AUC values for organ confined disease (OC), Seminal vesicle involvement (SVI) and Lymph node involvement (LNI) predictions were $82 \%, 80.5$ $\%$ and $71.4 \%$ respectively which show that the relationship between the predicted probabilities and observed results are good. This suggests that Partin's table would incorrectly classify only $18 \%, 19.5 \%$ and $28.6 \%$ of patients with respect to OC, SVI and LNI.

Histological grading of prostate biopsy specimens is one of the main determinants of prostate cancer treatment [6] and pathological Gleason score of RP specimen is a better predictor of biochemical recurrence than biopsy score [22,23]. Our study showed Gleason upgrading from biopsy to final pathology of $21.6 \%$. This is similar to the several retrospective studies which have demonstrated inadequate concordance rates between biopsy and pathological Gleason sum [2224]. Previous studies indicated that as many as $43 \%$ of men with low grade prostate cancer on biopsy will be finally diagnosed with high grade disease on radical prostatectomy [6,22-24]. Low Gleason score on pre treatment biopsy can lead to under treatment for clinically significant prostate cancer and likewise a high Gleason on biopsy can lead to over treatment. The Gleason down grading of RP specimen was $14.7 \%$ in our study.

Partin tables provide information only about the final pathological outcome and cannot predict the clinical outcome and therefore are not the ideal tools for further treatment planning. However, they can guide towards the type and extent of prostatectomy like nerve sparing to preserve the neuro-vascular bundle in patients with organ confined disease and need for routine lyphadenectomy or not.

Due to limitation of Partin tables, for the better counseling in the individual patients artificial neural networks (ANN) offer a more tailored treatment decision [25]. These ANN models were developed with reference to decision making process of the human brain and can improve the ability to predict organ confined and lymph node involvement more accurately in an individual than population based nomograms [26].

Our study has several limitations. It has a small sample size and was carried out in a tertiary care university teaching hospital setting rather than in a community based setting. The other validation studies included populations from various centers whilst ours in only a single centre study. The small sample size can over reflect the higher proportion of lymph node involvement and seminal vesicle involvement in our patient group. All the biopsy slides and pathology specimen were re-reviewed by a single histo-pathologist in a double blind manner which might decrease the inter observer variability in grading the tumor, however it does not reflect the real world practice.

To our knowledge, this is the first ever study which has determined the accuracy and discriminative properties of Partin tables from the South East Asian population, and therefore it can be used as a clinical decision making tool for patients with prostate cancer.

\section{Conclusions}

The 2007 Partin's table has a reasonable predictive value for the final histo-pathological features like organ confinement, seminal vesicle and lymph node involvement in our limited series. This predictive model can be used in Pakistani patients with carcinoma prostate with comparable accuracy.

\section{References}

1. Jemal A, Siegel R, Ward E, Murray T, Xu J, et al. (2006) Cancer statistics, 2006. CA Cancer J Clin 56: 106-130.

2. Naito S, Kuroiwa K, Kinukawa N, Goto K, Koga H, et al. (2008) Validation of Partin Tables and Development of a Preoperative Nomogram for Japanese Patients With Clinically Localized Prostate Cancer Using 2005 International Society of Urological Pathology Consensus on Gleason Grading: Data From the Clinicopathological Research Group for Localized Prostate Cancer. J Uro 180: 904-910.

3. Abrahamsson PA (2008) Prostate cancer and active surveillance. Front Radiat Ther Oncol 41: 1-6.

4. Walsh PC, Partin AW, Epstein JI (1994) Cancer control and quality of life following anatomical radical retropubic prostatectomy: results at 10 years. J Urol 152: 1831-1836

5. Partin AW, Mangold LA, Lamm DM, Walsh PC, Epstein Jl, et al. (2001) Contemporary update of prostate cancer staging nomograms (Partin tables) for the new millennium. Urology 58: 843-848.

6. Partin AW, Yoo J, Carter HB, Peason JD, Chan DW, et al. (1993) The use of prostate specific antigen, clinical stage and Gleason score to predict pathological stage in men with localized prostate cancer. J Urol 150: 110-114.

7. Bhojani N, Salomon L, Capitanio U, Suardi N, Shariat SF, et al. (2009)Externa validation of the updated partin tables in a cohort of French and Italian men. Int J Radiat Oncol Biol Phys 73: 347-352.

8. Epstein Jl, Allsbrook WC Jr, Amin MB, Egevad LL, ISUP Grading Committee (2005) The 2005 International Society of Urological Pathology (ISUP) Consensus Conference on Gleason Grading of Prostatic Carcinoma. Am J Surg Pathol 29: 1228-1242.

9. Shariat SF, Karakiewicz PI, Margulis V, Kattan MW (2008) Inventory of prostate cancer predictive tools. Curr Opin Urol 18: 279-296.

10. Oesterling JE, Brendler CB, Epstein JI, Kimball AW Jr, Walsh PC (1987) Correlation of clinical stage, serum prostatic acid phosphatase and preoperative Gleason grade with final pathological stage in 275 patients with clinically localized adenocarcinoma of the prostate. J Urol 138: 92-98.

11. Eskicorapci SY, Karabulut E, Türkeri L, Baltaci S, Cal C, et al. (2005) Validation of 2001 Partin tables in Turkey: a multicenter study. Eur Urol 47: 185-189.

12. Graefen M, Augustin H, Karakiewicz PI, Hammerer PG, Haese A, et al. (2003) Can predictive models for prostate cancer patients derived in the United States of America be utilized in European patients? A validation study of the Partin tables. Eur Urol 43: 6-11.

13. Makarov DV, Trock BJ, Humphreys EB, Mangold LA, Walsh PC, et al. (2007) Updated nomogram to predict pathologic stage of prostate cancer given prostate-specific antigen level, clinical stage, and biopsy Gleason score (Partin tables) based on cases from 2000 to 2005. Urology 69: 1095-1101.

14. Karakiewicz PI, Bhojani N, Capitanio U, Reuther AM, Suardi N, et al. (2008) External validation of the updated Partin tables in a cohort of North American men. J Urol 180: 898-903.

15. Zorn KC, Capitanio U, Jeldres C, Arjane P, Perrotte P, et al. (2009) Multiinstitutional external validation of seminal vesicle invasion nomograms: headto-head comparison of Gallina nomogram versus 2007 Partin tables. Int J Radiat Oncol Biol Phys 73: 1461-1467.

16. Robbins AS, Yin D, Parikh-Patel A (2007) Differences in prognostic factors and survival among white men and black men with prostate cancer, California, 1995-2004. Am J Epidemiol 166: 71-78

17. Tewari A, Horninger W, Pelzer AE, Demers R, Crawford ED, et al. (2005) Factors contributing to the racial differences in prostate cancer mortality. BJU Int 96: 1247-1252.

18. Heidenreich A, Aus G, Bolla M, Joniau S, Matveev VB, et al. (2008) EAU guidelines on prostate cancer. Eur Urol 53: 68-80. 
Citation: Nazim SM, Abbas F, Faruqui N, Islam M, Ahmad Z (2011) Validation of Updated Partin's Table in Pakistani Patients undergoing Radical Prostatectomy for Prostate Cancer J Cancer Sci Ther S1. doi:10.4172/1948-5956.S1-010

Page 5 of 5

19. Briganti A, Blute ML, Eastham JH, Graefen M, Heidenreich A, et al. (2009) Pelvic lymph node dissection in prostate cancer. Eur Urol 55:1251-1265

20. Briganti A, Chun FK, Salonia A, Gallina A, Farina E, et al. (2006) Validation of a nomogram predicting the probability of lymph node invasion based on the extent of pelvic lymphadenectomy in patients with clinically localized prostate cancer. BJU Int 98: 788-793.

21. Studer UE, Collette $L$ (2006) Morbidity from pelvic lymphadenectomy in men undergoing radical prostatectomy. Eur Urol 50: 887-889.

22. Epstein JI, Pizov G, Walsh PC (1993) Correlation of pathological findings with progression after radical retropubic prostatectomy. Cancer 71: 3582-3593.

23. King CR (2000) Patterns of prostate cancer biopsy grading: trends and clinical implications. Int J Cancer 90: 305-311.
24. Cookson MS, Fleshner NE, Soloway SM, Fair WR (1997) Correlation between Gleason score of needle biopsy and radical prostatectomy specimen: accuracy and clinical implications. J Urol 157: 559-562.

25. Lv DJ, Zhang Y, Wang XY, Guo XM, Wang CY (2009) Application of artificia neural network to diagnosis of prostate cancer. Beijing Da Xue Xue Bao 41 : 469-473.

26. Batuello JT, Gamito EJ, Crawford ED, Han M, Partin AW, et al. (2001) Artificial neural network model for the assessment of lymph node spread in patients with clinically localized prostate cancer. Urology 57: 481-485. 